\title{
TRANSVERSE FRICTIONS
}

\author{
By Brun. Winter, M.S.A.S.P., M.C.S.P., \\ Lecturer in Physiotherapy, University of Cape Town
}

Transverse friction is a massage technique, usually grouped with circular frictions, but it is not a Swedish petrissage technique.

\section{USES}

(1) Tendons-without sheaths, e.g. supraspinatus tendonitis (with no calcification); or with sheaths, e.g. tenosynovitis affecting the abductor pollicis longus and extensor pollicis brevis at the wrist.

(2) Ligaments-acute or chronic partial tears (sprains), e.g. the medial ligament of the knee, lateral ligament of the ankle.

(3) Muscles-minor tears (without gross haematoma), e.g. quadriceps.

\section{LOCALISATION AND ACCURACY}

These frictions can only affect superficial structures accessible to the finger, and that part covered by the frictioning finger. Hence it is essential that the tissue to be treated be superficial, isolated as the cause of pain, and the exact part affected pinpointed.

Frictions should then be applied to the whole of this area. It is therefore necessary for the physiotherapist to carry out the following examination of the patient before treatment is begun.

\section{EXAMINATION BY SELECTIVE TENSION}

The pain caused by the lesions named above can be reproduced or aggravated by putting tension on the affected structure. Tension is therefore put on to each tissue in turn, without involving the others. This isolates the affected structure.

If it is superficial, it is then palpated to find the point of maximal tenderness, compared to the normal side. This indicates the exact part affected, and this is where the frictions should be applied.

(1) Tendons. Tension is put on the affected tendon by resisted static contraction of the muscle with the joint in a neutral position. In tendonitis this contraction is painful but strong. Passive movements are full range and painless. All other resisted static contractions are strong and painless. Free active movement may be limited by pain. At the shoulder there may be a painful arc.

(2) Ligaments. Pain is produced when the sprained ligament is passively stretched. Passive movements may be limited in a disproportiontae way. Example: A sprain of

\section{Continued from page 4}

trauma. This has necessitated modification in physiotherapeutic management, the most noticeable modification being a decrease in the use of percussion. Basically, however, the aims and form of treatment by physiotherapy are much the same as they were when chest surgery was introduced.

\section{SUMMARY}

The place of physiotherapy in a thoracic surgical service has been defined. The physiotherapist should be a permanent member of and specific to a particular unit. Preoperative management of patients in whom pulmonary surgery is required and the schedule of early post-operative physiotherapy has been outlined, together with the place of physiotherapy in the management of a patient with a tracheostomy, on a respirator, and with a flail chest. Indications for bronchoscopy are given, and caution in the use of postural drainage in the circumstances of oesophagogastrectomy advised. The emphasis in post-operative physiotherapy is as the gentleness and frequency of treatment. the medial ligament of the knee-pain on passive valgus strain in extension, felt on the medial side. Some limitation of flexion but extension may be full range. Resisted static contractions of quadriceps and hamstrings strong and painless. Palpation reveals the site.

(3) Muscles. Resisted static contraction produces pain if a minor tear exists, but is strong. Passive movements are full and painless or may hurt a little when the muscle is fully stretched. All other resisted static contractions are strong and painless. Example: Partial tear of rectus femorispain on resisted static contraction, i.e. holding the knee extended against resistance; passive movements of the knee full and painless; some pain when the knee is fully flexed with hip extension; resisted static contractions of hamstrings strong and painless. Palpataion reveals the site.

\section{TECHNIQUE}

(a) Position of Patient: As for all treatments, the patient must be comfortable. For tendons without sheaths the part is positioned to make the tendon accessible. For tendons with sheaths, the part is positioned to keep the affected tendon/s on some degree of stretch in order to hold it firm so that the sheath can be moved across it. For ligaments the joint may be positioned in order to make the ligament accessible, put it on slight tension, or it may be put at the limit of the existing range (e.g. the medial ligament of the knee-frictions are applied with the knee flexed as far as possible). Muscles must be relaxed and supported in an inner range in order that the finger may reach "into" the fibres.

(b) Position of Physiotherapist: Frictions have to be maintained for at least 10 minutes, so she* should sit down. It is hard work for her hands, and alternate hands may be used, necessitating a change of position. She should be able to reach the affected structure without strain. Her arm should be positioned to obtain maximum sweep with minimum effort. Nails should be short, and not project beyond the finger pulp. The frictioning finger or thumb should be held slightly flexed or straight, but no joint should be hyperextended. It is common to use the index finger with the terminal interphalangeal joint flexed, reinforced by the middle finger (see diagram). The thumb may also be used, or two or three fingers next to each other may cover a wider area.

(c) Transverse sweep: This is the most important therapeutic movement. It is produced by movement of the physiotherapist's wrist, forearm, elbow or shoulder. Moving the finger alone is much too tiring. The direction should be at right angles to the striations of the structure being treated. The amplitude should be maximum allowed by the mobility of the patient's skin. It may follow the contour of a tendon in order to cover more than one aspect. The patient's skin moves with the physiotherapist's finger over the underlying structure. The patient's skin and the physiotherapist's finger should be dry and cool, otherwise slipping occurs, producing blisters or rubbing through the skin in a very short time. This may be achieved by washing in cool water and drying well. Methylated spirits may be applied to toughen the skin and dry it. For this reason no soaking or heat treatments or the application of liniments should precede frictions.

*Physiotherapist referred to as female for brevity. 


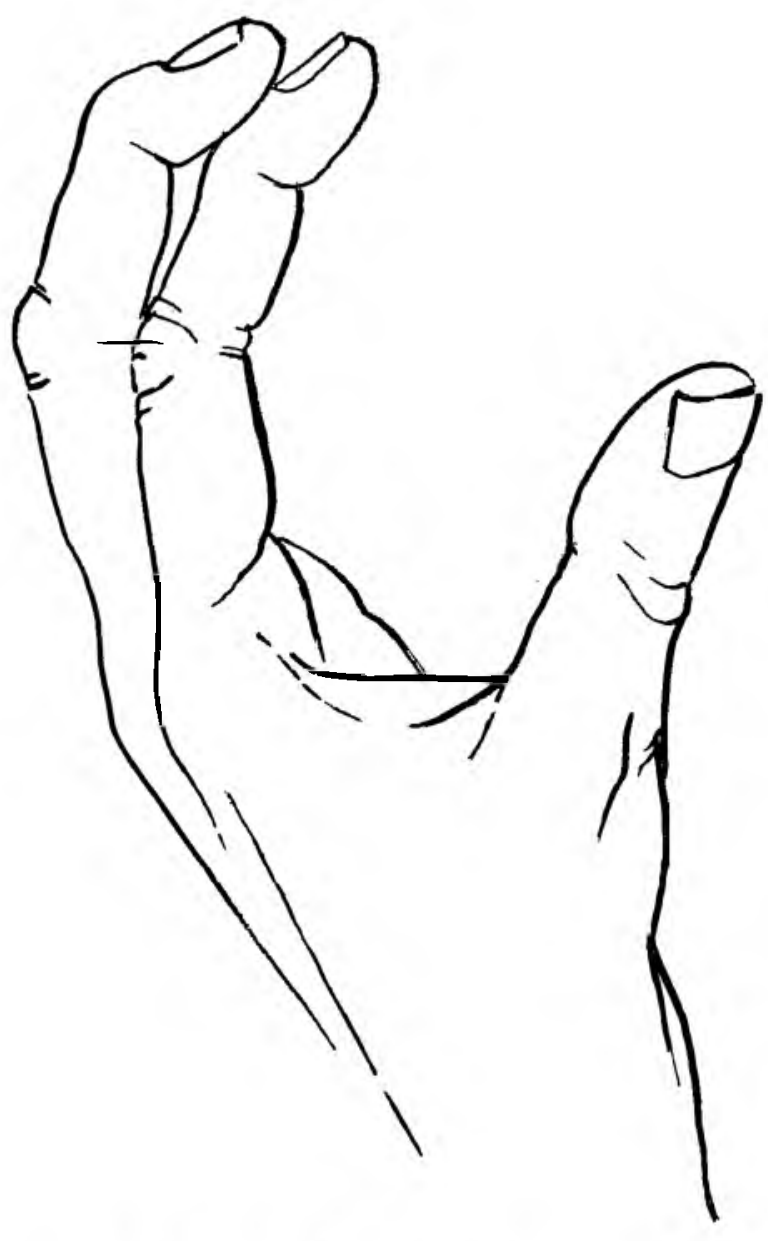

Position of hand for Transverse Frictions.

(d) Depth: Is achieved through pressure by the thumb or fingertip. The pressure is obtained by flexion of the finger and sometimes the weight of the physiotherapist's arm. It should be sufficient to ensure that no gliding occurs over the patient's skin, but it need not be a penetrating and painful pressure. In acute conditions, the depth is just sufficient to prevent slip, but the therapeutic sweep remains of maximum amplitude. It is therefore quite tolerable for the patient. After a few minutes the numbing effect occurs, and depth may then be increased. In this way the treatment, though never comfortable, is not painful, yet is deep enough to reach the affected structure and prevent adhesion formation. In chronic conditions where adhesions have to be broken down, depth can be increased because it is less painful.

(e) Duration: Treatment lasts from 10-20 minutes frictioning time. If the finger starts to slip and any danger of rubbing through occurs, it is better to stop frictions as once the skin is damaged, no further treatment can be done until it has healed.

\section{RECÛYERY}

From one to ten treatments may be required, given on alternate days or less often to give the local tenderness following frictions time to subside. Recovery should start after the first treatment, and the speed of recovery depends on several factors such as the accuracy and effectiveness of the frictions, the acuteness of the condition, the tissue being treated, and so on.

Criteria for Recovery. Only those tests which produced pain at the initial examination need be repeated before each treatment. Recovery is complete when these tests no longer hurt. Other signs of recovery are: pain more localised, i.e. not referred so far down the limb; pain no longer continuous; less pain on use or movement; increased joint range. Local tenderness must never be used to assess progress, as it is in fact increased by the frictions and may last up to five days after recovery. The patient should be told of this, and if deep frictions are done, of possible local bruising so that it does not cause alarm.

\section{EFFECTS}

(1) Mechanical Effect: In acute conditions, e.g. a minor muscle tear, tissue mobility may be maintained (adhesions prevented) without breaking down the fibres crossing the breach in a longitudinal direction. In chronic conditions tissue mobility may be restored (adhesions broken down), e.g. a medial ligament at the knee becoming adherent to the periosteum. These adhesions are not adequately prevented or broken down by passive or active movements alone hence their persistence. Active movement may in fact tear healing fibres causing further inflammation and adhesion formation.

(2) Circulatory Effect: An erythema which lasts for a short time, is visible after frictions. Superficial vasodilation therefore occurs due to the pressure, as the finger does not move over the skin. This is a reactive hyperaemia. It is thought that the same effect must also occur in the tissue treated, due to the pressure as well as the friction which occurs here. The vasodilation probably plays a part in the relief of pain, and helps the healing process in partial tears. For this reason it is not necessary to give additional vasodilating treatments (shortwave diathermy, infra-red radiation, ultrasound, Renotin ionization) after frictions.

(3) Analgesic Effect: This occurs during treatment, starting a few minutes after the commencement of frictions and lasting as long as the superficial erythema after frictions. The effect is so marked that the patient often says that the physiotherapist must have moved her finger, as it no longer feels tender. As soon as this occurs, the depth can be increased, enabling an effective yet tolerable treatment to be given, even in acute cases. This is the reason why additional analgesic treatments (Swedish massage, heat, anodal galvanism, ice) are not necessary before frictions are done. The period of analgesia is used to gain range, e.g. in acute ligamentous sprains, or procedures such as a Mill's manipulation may be carried out.

\section{CONCOMITANT TREATMENT}

(1) Tendons. Contraction of the affected muscle is painful and pulls on the lesion, especially if it is resisted. Exercises are therefore contraindicared in cases of tendonitis or tenosynovitis, including tennis elbow. They are not required to maintain or increase power as power is not lost, and function is normal as soon as the pain is abolished. Joint movement is not limited, so movements are not required to maintain or increase range. If it is feared that movement may become limited due to disuse, one full range passive or self-assisted movement per day, carried out by the patient, is adequate. The patient should be advised to avoid those movements that hurt, and partial rest may be achieved at the wrist by a small cock-up splint. It has been mentioned that treatments which warm and moisten the skin should never be applied before frictions, and are also unnecessary.

(2) Ligaments. In acute cases, frictions are followed by gentle passive movement in the direction of limitation, to increase range. In chronic cases the movement is short and 
sharp, to help break down adhesions. Range gained is maintained by instructing the patient to actively move the joint through full range several times a day.

At the knee, weakness and wasting of the vastus medialis may require strengthening by strong resisted isometric or isotonic work in as full extension as possible. These should be non-weightbearing until the ligament is no longer painful on being stretched, movement is full, and no effusion is present. Thereafter weight-bearing exercises and weightlifting through range may be done. If an effusion exists it needs no specific treatment. It may be controlled by the application of an elastic bandage, and disappears as soon as the ligament is healed and the vastus medialis is strong enough to stabilise the knee. Conditions causing gross effusion which interferes with range, are not treated by transverse frictions.

(3) Muscles. Frictions are followed by active nonweight-bearing exercises to regain full contractility and extensibility of the muscle. No resistance is applied as this is not only painful, but the tension created pulls on the healing scar. Intermittent ice towels may be applied while exercising. Graduated faradism may be used to gain full contractility if active movement fails.

\section{ALTERNATIVE TREATMENTS}

(1) Hydrocortisone injections (or $\frac{1}{2}$ per cent Procaine) given by the doctor, may be used if a structure is too deep for the finger to reach, or as an alternative to frictions. With hydrocortisone, the area is very painful for several hours after the injection, but when it wears off, improvement is evident.

(2) Counter-Irritation, e.g. Renotin ionization has been used, but it is a symptomatic and not curative treatment. In the author's opinion, it is not effective.

(3) Heat produces temporary analgesia and diffuse vasodilation, not as effective as that produced by transverse frictions.

(4) Intermittent applications of iced towels are of benefit in acute sprains and muscle tears, and exercises are done concomitantly.

(5) Ultrasound is, in the author's opinion, not as effective as transverse frictions.

\section{CONTRA-INDICATIONS}

1. Any structure or condition not mentioned above.

2. If previous frictions have damaged the skin due to poor technique, no further frictions to that area can be done until the skin has healed.

3. Some patients' skins react out of all proportion to the irritant. This may happen in one in two hundred patients.

Transverse frictions (sometimes incorrectly named cross frictions) have been used, misused and even abused for years now. As usual, it is a very effective treatment if carried out correctly and for the corrrect conditions. It cannot beexpected to be effective if not carried out accurately or correctly or to tissues too deep to reach, or for the wrong condition.

Some physiotherapists feel it is a cruel, painful treatment, but this opinion is based on inadequate knowledge of the technique and effects. It is hoped that this article may help to put the use of transverse frictions in the right perspective.

\section{ACKNOWLEDGEMENTS}

Thanks are due to Dr. J. Cyriax and Miss J. Hickling for personal instruction in this method of treatment, and to Miss J. Gibson for the diagram.

\section{NATIONAL COUNCIL}

\section{for the \\ CARE of CRIPPLES}

\section{ANNOUNCEMENT}

"Cripple Care" today embraces every aspect of rehabilitation. It starts with the discovery of crippling conditions, whether these be congenital or caused by injury or disease; it involves treatment, transport, training, accommodation and employment; it concerns Government Departments, Provincial Administrations, Local Authorities and Voluntary Organisations; it affects hospitals, homes and human beings; it caters for cripples of all ages and all races.

The Easter Stamp Fund for Cripples provides the money which the National Council for the Care of Cripples in South Africa uses to advance all facets of cripple care work.

Easter Stamps are on sale throughout the month of March. By supporting the Easter Stamp Fund EVERYBODY can help restore cripples to useful independence. Cripples don't want charity-give them a chance to become taxpayers.

Please support the EASTER STAMP FUND and help cripples to help themselves.

\section{A. C. MILLER \& CO. ORTHOPAEDIC MECHANIIIANS}

Manufacturers and Suppliers of:

ORTHOPAEDIC APPLIANCES, ARTIFICIAL LIMBS, TRUSSES, SURGICAL CORSETS, URINALS, ARCH SUPPORTS, COLOSTOMY BELTS, ELASTIC STOCKINGS, ANKLE GUARDS, WRIST GUARDS, ELBOW GUARDS, KNEE GUARDS, LIGHT DURAL CRUTCHES FOR CHILDREN, WOODEN CRUTCHES, AND METAL ELBOW CRUTCHES.

Phone 23-2496 P.O. Box 3412 312 Bree Street, Johannesburg 